\title{
Novel Butterfly Slot Based Chipless RFID Tag
}

\author{
Muhammad Ali RIAZ ${ }^{1}$, Yassin ABDULLAH ${ }^{1}$, Humayun SHAHID $^{1}$, Yasar AMIN ${ }^{1}$, Adeel AKRAM ${ }^{1}$, \\ Hannu TENHUNEN ${ }^{2,3}$ \\ ${ }^{1}$ ACTSENA Research Group, University of Engineering and Technology, 47050-Taxila, Pakistan \\ 2 iPack VINN Excellence Center, Royal Institue of Technology, SE-16440, Stockholm, Sweden \\ 3 TUCS, University of Turku, Turku-20520, Finland \\ ali.riaz@uettaxila.edu.pk
}

Submitted May 5, 2018 / Accepted June 19, 2018

\begin{abstract}
A compact chipless RFID tag with robust readable features is presented in this paper. The tag is made up of novel concentric butterfly slot resonators. Bit data is encoded in the frequency signature of the tag. Each slot corresponds to a resonance peak representing a bit ' 1 ', whereas an absence of the peak signifies a bit ' 0 '. Proposed resonator design demonstrates insensitivity to different polarization and incident angles of the linearly polarized impinging electromagnetic wave. The tag operates in the frequency band of 4.7-9.7 GHz, limited within the license-free ultra wide-band. Rogers RT/duroid ${ }^{\circledR} 5880$ substrate is used to realize a 10-bit capacity design that spans $14 \times 14 \mathrm{~mm}^{2}$ resulting in a bit density of $5.1 \mathrm{bits} / \mathrm{cm}^{2}$.
\end{abstract}

\section{Keywords}

Radio frequency identification (RFID), chipless tag, electromagnetic signature, Radar Cross-Section (RCS)

\section{Introduction}

Radio frequency identification (RFID) is a technique used to identify unique objects using radio frequency waves in a wireless medium [1]. A standard RFID tag primarily consists of a silicon chip (IC) and a transceiving antenna. Tag information is stored in the chip that modifies the received interrogating signal based on coded information and sends it back to the reader. An antenna is incorporated with the IC to facilitate efficient reception and transmission of wireless RF signals [2]. However, the antenna adds an additional bulk to the overall tag size [3], spanning over a substantial surface area of the tag as compared to that of the IC. Moreover, the use of IC serves as a hindrance to bringing down the cost per tag [4]. A minimum power of $15 \mathrm{dBm}$ is required to operate the IC, and its terminal impedance also needs to be perfectly matched to that of the antenna that further complicates the operating model [5]. Chipless RFID tags are proposed to alleviate the economic constraint of RFID tags by eliminating the need of the IC [6-10]. Furthermore, frequency selective surface (FSS) based chipless RFID tags [11] neither require an integrated antenna nor exhibit minimum power require- ments: making the tag more compact and power efficient. Chipless RFID tags are termed as the barcode of the future [6], [7]. The manufacturing process of the tag is fairly simple and similar to that of the optical barcode, since the tag can be produced by using printing processes. Unlike barcodes, chipless RFID tags can efficiently communicate over non-line of sight scenarios and offer higher read range.

Chipless RFID tags are primarily classified into timedomain and frequency domain based tags. Although chipless RFID tags are not very common from a commercial point of view, the only ones available are Surface Acoustic Wave (SAW) tags [12-14]. SAW principally converts the electromagnetic waves into many slower acoustic components and makes use of piezoelectric components. SAW tags are time domain based tags having relatively low data capacity [13] and involve a complex sub-micron lithographic process for manufacturing [15]. Another proposed timedomain RFID tag is the transmission delay line tag [16] that works on the principle of producing time delay in the received signal through an inductor-capacitor (LC) transmission line elements. The delay profile in the time domain is then used to identify the data stored in the tag. Time delay tags are typically larger in size in comparison with other chipless RFID tags [16-18].

Frequency domain chipless RFID tags are designed to encode bit sequences in the frequency domain through presence or absence of resonant peaks, signified as data bits. These are further categorized into retransmission and RCS-based tags. In retransmission based tags, a multiresonant RF circuit is used to encode bit sequences with a pair of identical cross-polar antennas for receiving and transmitting electromagnetic waves [19]. Unlike circuit-based tags, RCS-based tags do not require antennas to operate. These tags are made up of multi-resonant frequency selective surfaces to achieve substantial absorption of EM waves at resonant frequencies [20]. The multi-resonant elements are usually placed in a nested manner to efficiently utilize the surface area. Although RCS based chipless RFID tags offer low-cost solution for mass production, enhancement of bit capacity and density are constrained due to multiple reasons, such as: limited operating frequency band [22], inter-resonator 
coupling [15], [21], and presence of higher order harmonics [23]. Although recently proposed RCS based chipless RFID tags [15, 20,24] are compact having low production cost, spectral efficiency has not been taken into consideration. Operating over a wide band at higher frequencies requires advanced reading system, resulting in higher reader setup cost. On the other hand, a narrow band tag design at low frequencies serves as a constraint to achieving smaller tag size, since low-frequency resonating elements are larger in size and must be placed at a certain gap to minimize mutual coupling effects. Moreover, second and third harmonics of low-frequency resonating elements also hamper the utilization of a wider band.

In this paper, a novel butterfly shaped slot resonator is proposed. Furthermore, a compact chipless RFID tag having a size of $1.96 \mathrm{~cm}^{2}$ is designed using a set of these resonators, providing a spectral efficiency of 2 bits $/ \mathrm{GHz}$ while maintaining a competitive bit density of $5.1 \mathrm{bits} / \mathrm{cm}^{2}$ and a capacity of 10 bits. The tag operates in the frequency band of 4.7-9.7 GHz and its performance is analyzed over different polarization and incident angles of the impinging electromagnetic wave. Having a capacity of 10 bits the design is realized using Rogers RT/duroid ${ }^{\circledR} 5880$ substrate.

\section{Resonant Element Design}

Construction of polarization insensitive geometric structure and its parameters are illustrated in Fig. 1. A pair of similar ellipses is used to design the resonator. Each ellipse is defined by two parameters: the large diameter $D_{\mathrm{a}}$ and a small diameter $D_{\mathrm{b}}$. A rotation of $+45^{\circ}$ and $-45^{\circ}$ in the XY-plane is applied to each ellipse, and the two elements are finally added to obtain the resulting butterfly shape.

The structure is realized on a $14 \times 14 \mathrm{~mm}^{2}, 0.508 \mathrm{~mm}$ thick, Rogers RT/duroid ${ }^{\circledR} 5880$ substrate as a slot resonator as illustrated in Fig. 2. A slot width is introduced, keeping outer boundary of the elliptical slot defined by $D_{\mathrm{a}}$ and $D_{\mathrm{b}}$. The physical parameters of the tag are optimized to achieve substantial electromagnetic absorption near $5 \mathrm{GHz}$ without repetitions in the FSS structure while having minimal $2^{\text {nd }}$ and $3^{\text {rd }}$ harmonic resonances. The optimized values for $D_{\mathrm{a}}, D_{\mathrm{b}}$ are obtained as $16 \mathrm{~mm}$ and $10.53 \mathrm{~mm}$ respectively, having a slot width, $w$ of $0.23 \mathrm{~mm}$.

Horizontally polarized electromagnetic wave is used for analyzing the electromagnetic performance of the tag having a single sample of the proposed resonator using CST MICROWAVE STUDIO ${ }^{\circledR}\left(\right.$ CST $^{\circledR}$ MWS $\left.^{\circledR}\right)$. The radar cross section (RCS) of the tag is shown in Fig. 2, displaying a resonance at $5.1 \mathrm{GHz}$ as its fundamental frequency component. It is worth noting that the proposed resonator has negligible $2^{\text {nd }}$ and $3^{\text {rd }}$ harmonics since no major resonances are observed near $10.2 \mathrm{GHz}$ and $15.3 \mathrm{GHz}$. Although, a band of 4.7-9.7 GHz is utilized in this work to achieve higher spectral efficiency, the capacity of the proposed tag in Sec. 3 can be enhanced by nesting high-frequency resonators at the cost of much sophisticated reader setup.

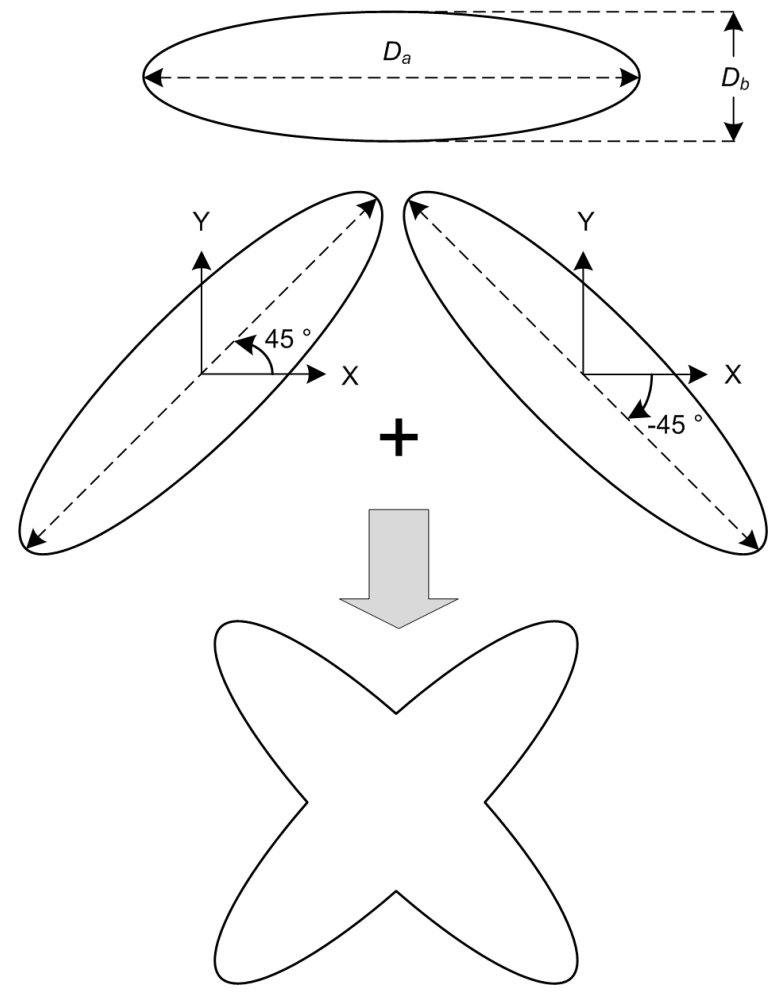

Fig. 1. Geometric construction of resonating element.

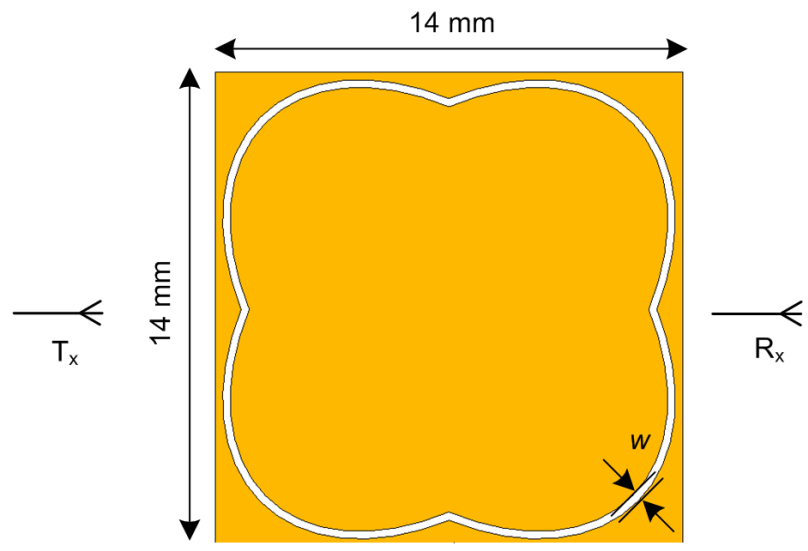

(a)

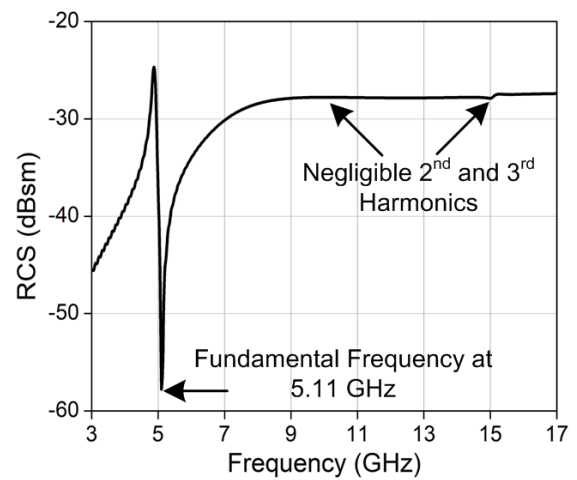

(b)

Fig. 2. Butterfly shaped slot resonator (a) geometry and (b) its frequency response. 
Surface current distribution of the slot resonator at its resonant frequency of $5.11 \mathrm{GHz}$ is depicted in Fig. 3 for a horizontally polarized incident plane wave. A high surface current is observed around the top and bottom sides of the resonator that signifies inductive behavior, whereas low surface current density areas indicate capacitive profile. A standing wave mode is created at resonant frequency through the interplay of these capacitive and inductive components as shown in surface current distribution, and electric field intensity. Moreover, $\lambda / 4$ resonance is validated through simulation using $\left(\mathrm{CST}^{\circledR} \mathrm{MWS}^{\circledR}\right)$ as illustrated by the surface current distribution as shown in Fig. 3 (a).

The resonator design primarily offers compact features and geometrical symmetry owing to high bit density and orientation independent operability. Moreover, nesting of additional multi-resonant elements is possible within the same tag size. Having five reflection and four rotational symmetries as shown in Fig. 4, the symmetric, curved shape of the resonator not only reduces the harmonic components but also makes it polarization insensitive.

The electromagnetic performance of the tag having a single butterfly shaped resonator is analyzed at a variety of polarization angles using $\mathrm{CST}^{\circledR} \mathrm{MWS}^{\circledR}$. Although, the overall RCS magnitude response of the tag moves downwards with the change in polarization angle, the resonance frequency remains almost unchanged and no spurious peaks are observed. Since the structure is $90^{\circ}$ - rotationally symmetric, similar frequency response is observed for polarization angles of $0^{\circ}$ and $90^{\circ}$.

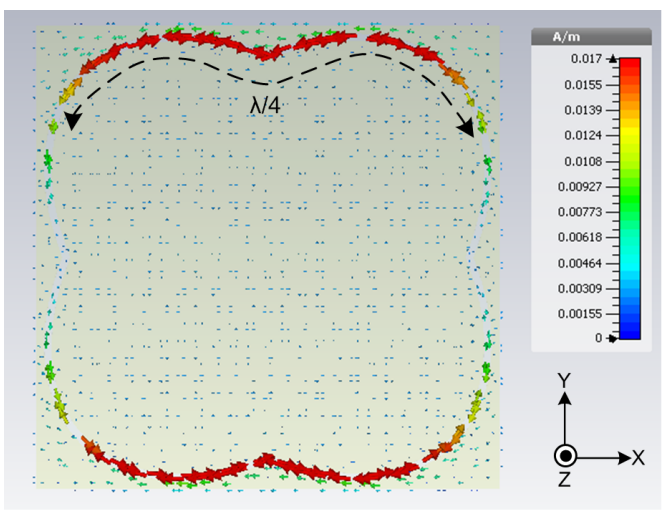

(a)

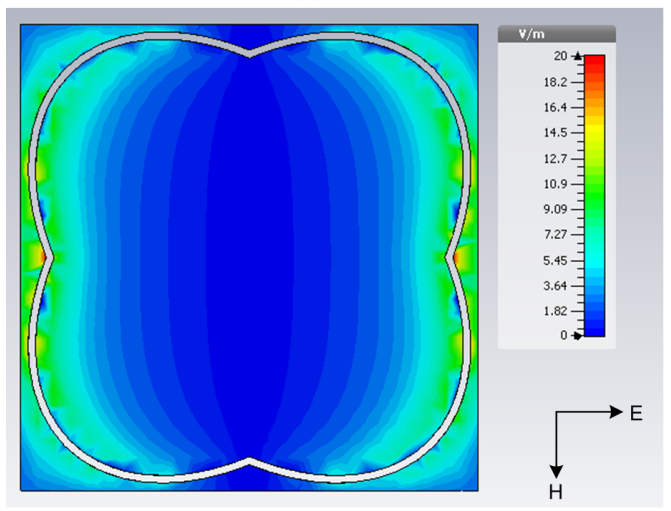

(b)

Fig. 3. (a) Surface current distribution and (b) electric field intensity in the plane of resonator at $5.11 \mathrm{GHz}$

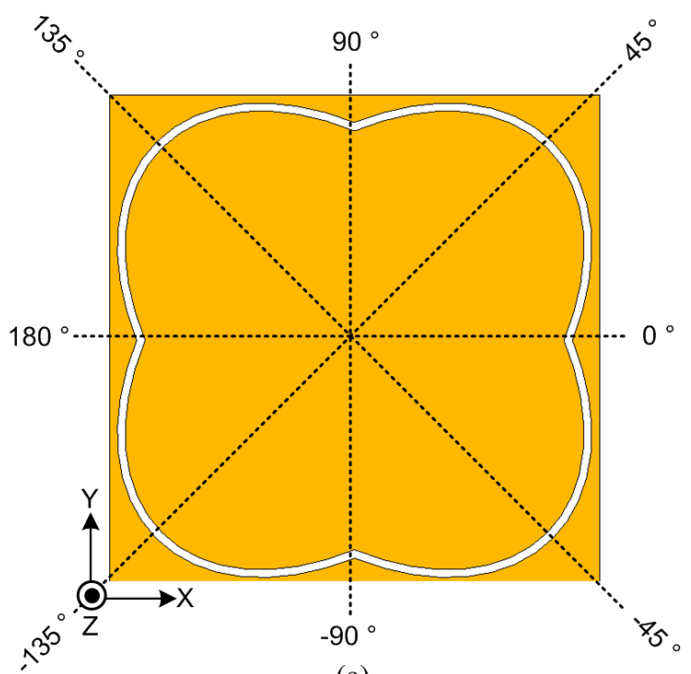

(a)

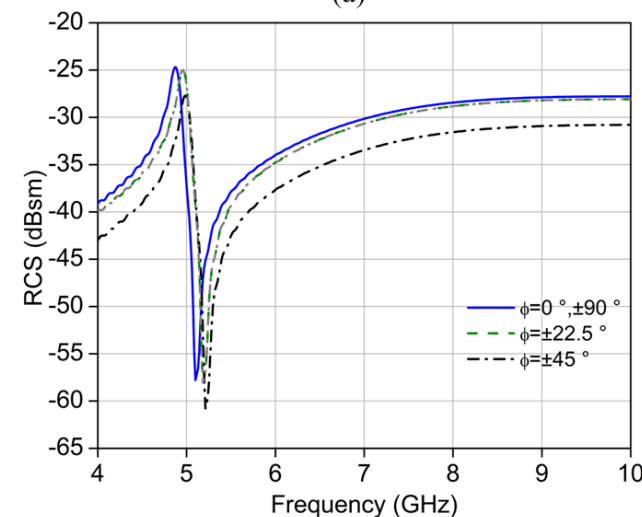

(b)

Fig. 4. (a) Symmetry of the resonator and (b) RCS response at different polarization angles.

\section{Overall Tag Design}

Nesting of additional butterfly resonators is introduced in the tag presented in Sec. 2 to obtain multiple resonances while maintaining a compact footprint. The resonators are placed at a gap, $g=0.23 \mathrm{~mm}$ which is same as that of the slot width $w$. Ground plane on the bottom side of the tag is not used since the design is slot resonator based. Negative etching has been applied to obtain the overall resonating structure.

Size comparison of a prototype chipless RFID tag with a euro coin having ten nested resonators is shown in Fig. 5. The outermost slot element resonates at a minimum frequency, corresponding to least significant bit (LSB). Whereas, the most significant bit (MSB) is related the smallest slot element that resonates at maximum frequency. Although additional resonating elements may be added on either side, more surface area of the tag is required to accommodate larger elements: resulting in a decline of bit density. Moreover, an introduction of smaller elements results in higher resonant frequencies, decreasing the spectral efficiency of the tag. 


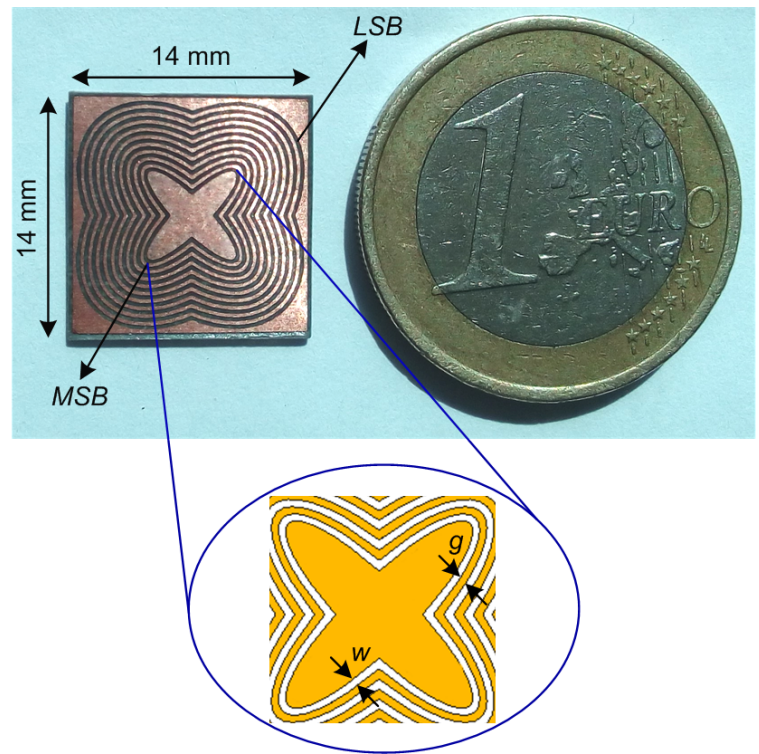

Fig. 5. 10 bit chipless RFID tag.

Exact dimensions of resonators with reference to each bit position are provided in Tab. 1. These dimensions are parametrically chosen to limit the operating band of the tag within 5-10 GHz for ensuring a combination of high spectral and bit density. Bit encoding is achieved through addition and removal of slot resonators. Removal of each slot resonator corresponds to the absence of its corresponding resonance peak in the RCS of the tag and vice versa. Presence of each resonance signifies a bit ' 1 ', whereas, its absence is considered as a bit ' 0 '.

\begin{tabular}{|c|c|c|c|c|c|}
\hline Bit Position & LSB & $\mathbf{1}$ & $\mathbf{2}$ & $\mathbf{3}$ & $\mathbf{4}$ \\
\hline $\boldsymbol{D}_{\mathbf{a}}[\mathrm{mm}]$ & 16 & 15.08 & 14.16 & 13.24 & 12.32 \\
\hline $\boldsymbol{D}_{\mathbf{b}}[\mathrm{mm}]$ & 10.53 & 9.61 & 8.69 & 7.77 & 6.85 \\
\hline Bit Position & $\mathbf{5}$ & $\mathbf{6}$ & $\mathbf{7}$ & $\mathbf{8}$ & MSB \\
\hline $\boldsymbol{D}_{\mathbf{a}}[\mathrm{mm}]$ & 11.4 & 10.48 & 9.56 & 8.64 & 7.72 \\
\hline $\boldsymbol{D}_{\mathbf{b}}[\mathrm{mm}]$ & 5.93 & 5.01 & 4.09 & 3.17 & 2.25 \\
\hline
\end{tabular}

Tab. 1. Dimensions of slot resonators.

\section{Results and Discussion}

The proposed tag prototype variants with all ones, all zeros, and random sequence are shown in Fig. 6 with their respective computed electromagnetic response obtained through $\mathrm{CST}^{\circledR}$ MWS ${ }^{\circledR}$. The RCS response of all one's prototype demonstrates ten distinct resonances, using the same amount of nested elements. A random sequence is obtained through removal of resonators resulting in the absence of corresponding resonant peaks. However, a slight shift in each resonant frequency is observed for different combinations, because of its dependence on the physical parameters of the corresponding resonator, and the elements in its surroundings. The resulting resonant frequency is hence produced through a combination of resonator geometry and mutually coupled effects introduced by surrounding resonators. Each resonance is assigned a spectral neighborhood of $300 \mathrm{MHz}$

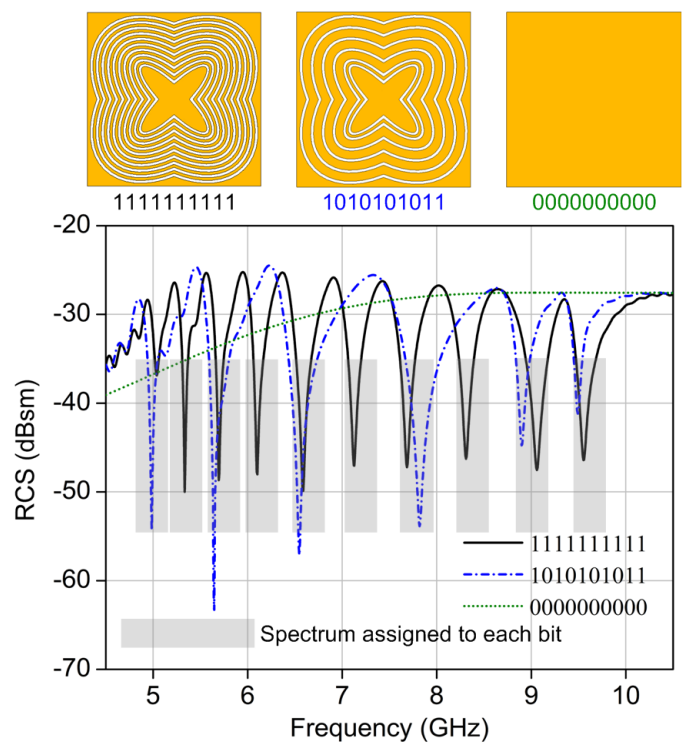

Fig. 6. Computed RCS response for different bit sequences.

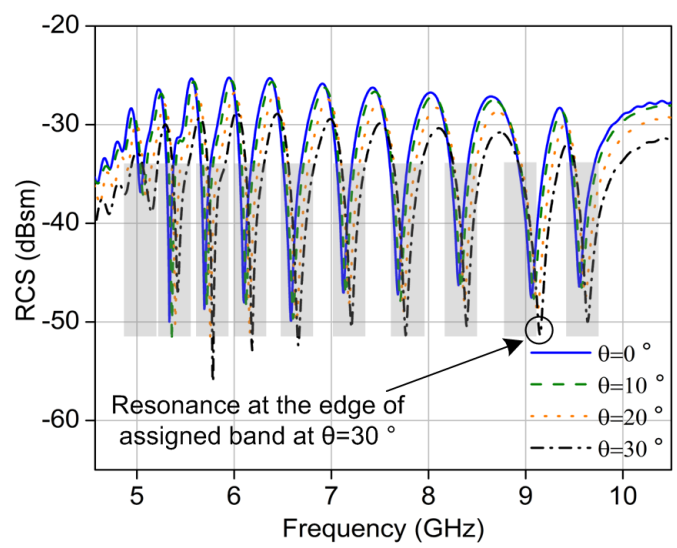

Fig. 7. Oblique incidence performance.

to allow for accurate detection of bit positions under frequency shift circumstances, which is mentioned in Fig. 6 as a shaded region. A gap of $0.23 \mathrm{~mm}$ is chosen between resonators to ensure minimal coupling effects while maintaining compact size of the tag. The design offers avoidance of overlapping resonances, limiting them within the corresponding spectral neighborhood.

The oblique incidence electromagnetic performance of the tag shown in Fig. 7 is computed using CST $^{\circledR}$ MWS $^{\circledR}$. This demonstrates the readability of the tag in a leaning position with respect to the impinging electromagnetic wave. A shift in resonant frequencies with a reduction in RCS magnitude is observed as the tag is tilted. Since the band of operation is limited for each bit, it needs to be ensured that frequency shifts at oblique incident angles remain within these limits. According to the computed results, the tag operates at incident angles of up to $30^{\circ}$. This limitation is primarily introduced by the resonant frequency around $9 \mathrm{GHz}$, that lies at the edge of its corresponding frequency band as depicted in Fig. 7. 
A standard measurement setup procedure is utilized that is mentioned in detail elsewhere [25]. Measurements for the tag have been performed using a two-port vector network analyzer (VNA) R\&S ZVL-13, and a pair of identical linearly polarized horn antennas. Continuous wave stepped frequency technique is employed to detect the backscattered power from the tag, that is recorded throughout the frequency sweep process. For the band of $4.7-10.7 \mathrm{GHz}$, the VNA transmits $0 \mathrm{dBm}$ of power. The tag is placed in the far-field region, at a distance of $35 \mathrm{~cm}$ from the interrogating antennas. Two reference measurements, one with no tag, and one with a full-metallic plate of comparable dimensions with known RCS are carried out prior to commencing with testing with the tag prototype. The procedure is concluded with the measurements for the tag prototype in place.

A comparison between measured and computed results for the tag having all 1's configuration is reported in Fig. 8. Although, a slight shift in resonant frequencies is observed, which is due to the structural infirmity of realized tag introduced through the fabrication process. All resonances are distinctly observable with good absorption levels. This demonstrates that the proposed FSS tag structure needs not to be repeated to achieve satisfactory absorption at resonant frequencies resulting in a compact design. Furthermore, no spurious peaks are observed in either computed or experimental results: validating 1:1 correspondence between resonators and data bits.

Figure 9 illustrates the measured and computed response of the proposed tag with random bit sequence. Removal of the slot resonators results in complete disappearance of corresponding resonance, depicting the bit sequence reconfigurability of the tag. However, an increase in electromagnetic absorption level of low-frequency slot resonators is observed in the absence of their neighboring elements. Despite a slight shift of the resonant frequencies due to change in the mutual coupling, no spurious peak or significant variation, such as flattening of neighboring peaks is observed.

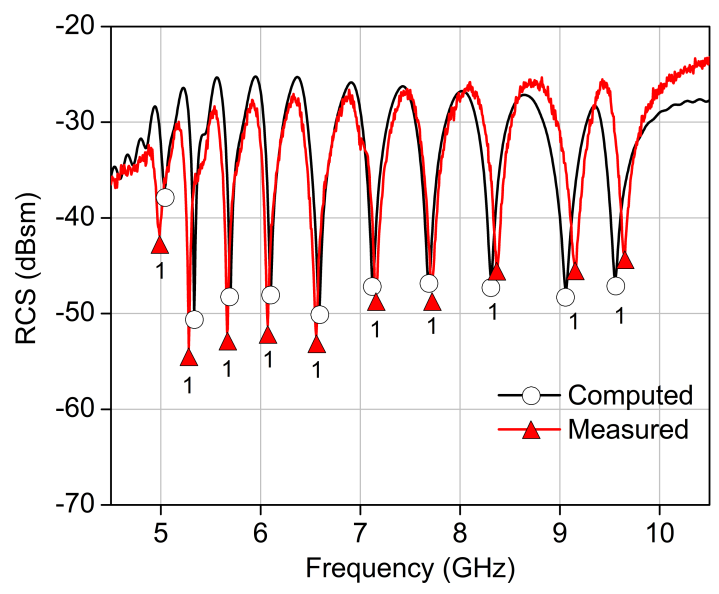

Fig. 8. Measured and computed response for all 1's sequence.
The measured RCS response of the tag for different bit sequences is shown in Fig. 10. As discussed previously, a shift in resonant frequencies is observed primarily due to two reasons: 1) difference of mutual coupling among resonators for a variety of bit combinations and 2) slight impairments in the resonating structure introduced by fabrication process. The tag design caters both challenges, since the measured resonant frequencies lie within their corresponding frequency spectrum. Each resonance peak can be automatically detected using the difference between local maxima and minima of the measured RCS profile within the assigned frequency band.

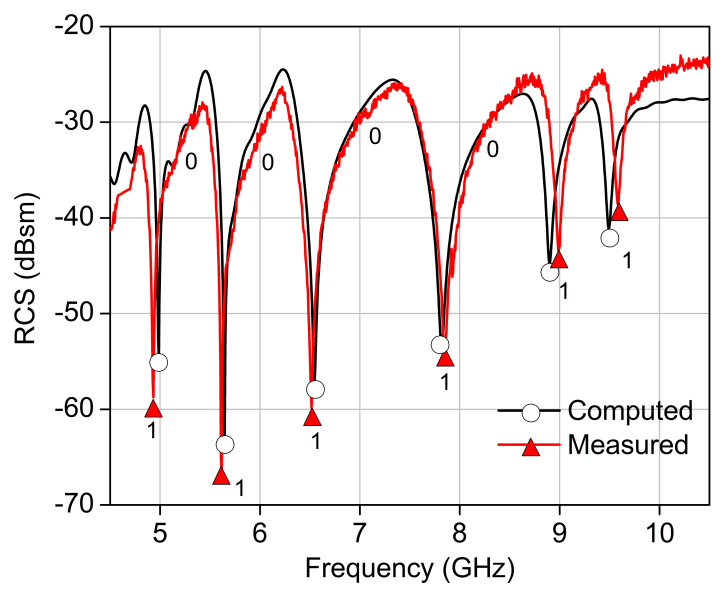

Fig. 9. Measured and computed response for random sequence.

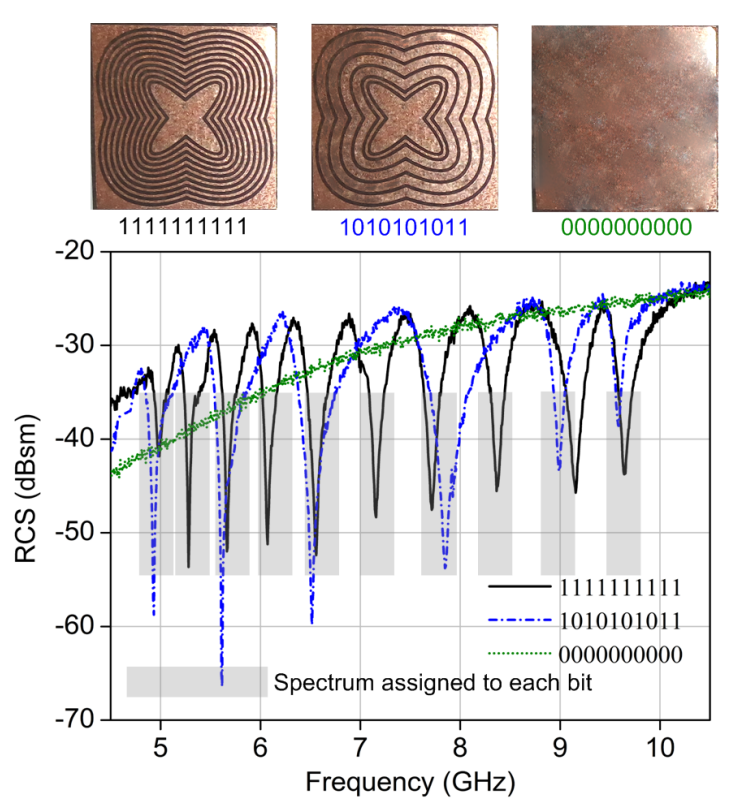

Fig. 10. Measured RCS response for different bit sequences. 
Figure 11 shows the measured RCS of the tag at a variety of oblique incident angles in comparison with the computed RCS. Although the resonance peaks are uniquely identifiable, they must lie within their corresponding frequency band for accurate bit detection. It is observed that measured frequency shifts due to the slanted orientation of the tag can be accommodated for incident angles of up to $20^{\circ}$. However, at $30^{\circ}$ orientation, the measured resonance near $9 \mathrm{GHz}$ slips out of its corresponding frequency band. Although this work utilizes same bandwidth for all resonances, this limitation maybe alleviated through assigning wider bands for high frequency peaks.

The measured RCS response at a variety of polarization angles is shown in Fig. 12. It is evident that the resonant frequencies and magnitude have very slight variations. Due to the $90^{\circ}$-rotational symmetry of the resonator design, its response remains the same at $0^{\circ}$ and $90^{\circ}$ polarization angles. For chipless RFID tags, this is a highly sought-after feature in terms of its practical utilization. The resonator design is hence polarization insensitive.

A comparison of the proposed work with recently proposed FSS based chipless RFID tags is shown in Tab. 2. The tag offers 10 bits of information within a compact size of $1.96 \mathrm{~cm}^{2}$ operating within the frequency band of 4.7-9.7 GHz. Typically, tag designs are oriented towards achieving higher bit density (bits/ $\mathrm{cm}^{2}$ ) to pack higher information within a compactly sized tag. However, spectral efficiency (bits/GHz) is often not taken into consideration, that may result in high reader setup cost. Although, hexagonal loop resonator based tag [24] offers a slightly higher bit density, its spectral efficiency is less than half to that of the proposed design. Furthermore, it requires a wide band of $4-19 \mathrm{GHz}$ that requires a more sophisticated setup to operate. Hence, the proposed design offers a combination of both: high bit, and high spectral efficiency with robust readability.

\begin{tabular}{|c|c|c|c|}
\hline Resonator Shape & $\begin{array}{c}\text { Tag Size } \\
{\left[\mathrm{cm}^{2}\right]}\end{array}$ & $\begin{array}{c}\text { No. of } \\
\text { Bits }\end{array}$ & $\begin{array}{c}\text { Frequency } \\
\text { Band [GHz] }\end{array}$ \\
\hline Butterfly (this work) & 1.96 & 10 & $4.7-9.7$ \\
\hline T-shape [15] & 2.25 & 10 & $6.0-15.0$ \\
\hline Hexagon [24] & 2.30 & 14 & $4.0-19.0$ \\
\hline Circular Loop [26] & 2.36 & 09 & $6.0-14.0$ \\
\hline Triangular [20] & 8.00 & 10 & $4.0-11.0$ \\
\hline Rectangular [11] & 9.00 & 05 & $2.0-8.0$ \\
\hline Resonator Shape & $\begin{array}{c}\text { Bit } \\
\text { Density } \\
{\left[\text { bits/cm }{ }^{2} \text { ] }\right.}\end{array}$ & $\begin{array}{c}\text { Spectral } \\
\text { Effi- } \\
\text { ciency } \\
{[\text { bits/GHz] }}\end{array}$ & $\begin{array}{c}\text { Polarization } \\
\text { Insensitiv- } \\
\text { ity }\end{array}$ \\
\hline Butterfly (this work) & 5.10 & 2.00 & Yes \\
\hline T-shape [15] & 4.44 & 1.11 & No \\
\hline Hexagon [24] & 6.08 & 0.93 & Yes \\
\hline Circular Loop [26] & 3.80 & 1.12 & Yes \\
\hline Triangular [20] & 1.21 & 1.42 & No \\
\hline Rectangular [11] & 1.23 & 0.83 & Yes \\
\hline
\end{tabular}

Tab. 2. Comparison with existing chipless RFID tag designs.

\section{Conclusions}

A novel butterfly resonator based chipless RFID tag has been proposed. The compact tag carrying 10 bits comprises of non-repetitive $14 \times 14 \mathrm{~mm}^{2}$ slot based FSS structure offering 1:1 bit to resonator correspondence. The tag is realized using a $0.508 \mathrm{~mm}$ thick, Rogers RT/duroid ${ }^{\circledR} 5880$ substrate. Encoding of bit sequences is achieved through addition and removal of nested elements resulting in a presence or absence of the correlated resonant peak in the frequency domain. The design offers robust readable features including polarization insensitivity and operability at oblique incidence angles extending up to $30^{\circ}$ of the impinging electromagnetic wave. The design operates within the frequency band of $4.7-10.7 \mathrm{GHz}$ and offers a combination of high bit density and spectral efficiency of $5.1 \mathrm{bits} / \mathrm{cm}^{2}$ and $2 \mathrm{bits} / \mathrm{GHz}$ respectively.

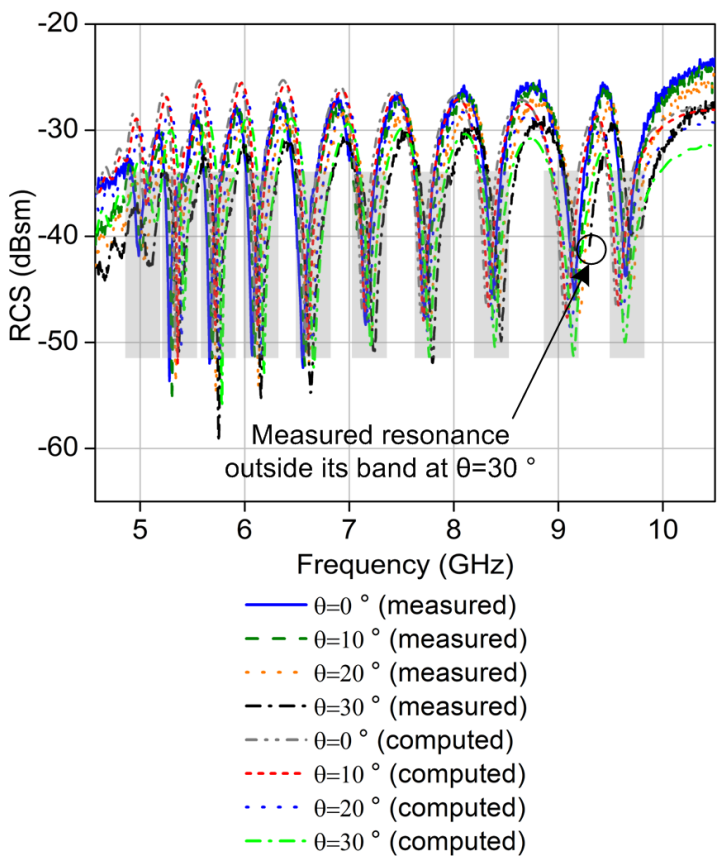

Fig. 11. Measured and computed RCS response at oblique incidence.

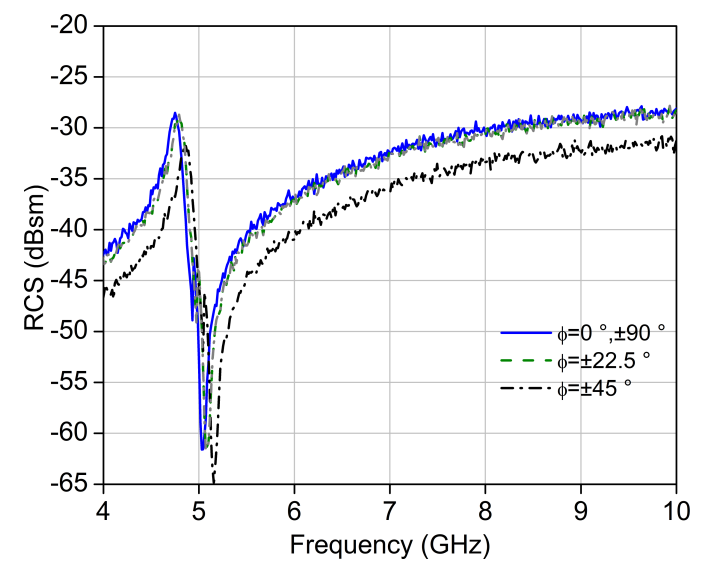

Fig. 12. Measured RCS response at different polarization angles. 


\section{Acknowledgments}

This work is financially backed by UET, Taxila via the ACTSENA research group funding. Furthermore, National Institute of Electronics, Islamabad is acknowledged for extensive support during prototype fabrication phase.

\section{References}

[1] FINKENZELLER, K. RFID Handbook: Fundamentals and Applications in Contactless Smart Cards, Radio Frequency Identification and near Field Communication. 3rd ed., rev. New York (USA): Wiley, 2010. ISBN: 9780470665121

[2] KHAN, U. H., ASLAM, B., KHAN, J., et al. A novel asterisk-shaped circularly polarized RFID tag for on-metal applications. Applied Computational Electromagnetic Society Journal, 2016, vol. 31, no. 9, p. 1035-1042. ISSN: 1054-4887

[3] DEY, S., SAHA, J. K., KARMAKAR, N. C. Smart sensing: Chipless RFID solutions for the internet of everything. IEEE Microwave Magazine, 2015, vol. 16, no. 10, p. 26-39. DOI: 10.1109/MMM.2015.2465711

[4] KARMAKER, N. C. Tag, You're it radar cross section of chipless RFID tags. IEEE Microwave Magazine, 2016, vol. 17, no. 7, p. 64-74. DOI: 10.1109/MMM.2016.2549160

[5] KHAN, U. H., RASHEED, H., ASLAM, B., et al. Localization of compact circularly polarized RFID tag using ToA technique. Radioengineering, 2017, vol. 26, no. 1, p. 147-153. DOI: $10.13164 /$ re.2017.0147

[6] PRERADOVIC, S., KARMAKAR, N. C. Chipless RFID: Bar code of the future. IEEE Microwave Magazine, 2010, vol. 11, no. 7, p. 87-97. DOI: 10.1109/MMM.2010.938571

[7] PRERADOVIC, S., KARMAKAR, N. C. Multiresonator-Based Chipless RFID. 1st ed., rev. New York (USA): Springer, 2012. ISBN: 9781461420958

[8] PERRET, E. Radio Frequency Identification and Sensors: From RFID to Chipless RFID. New York (USA): Wiley, 2014. ISBN: 9781119054016

[9] REZAIESARLAK, R., MANTEGHI, M. Chipless RFID. Switzerland: Springer, 2015. ISBN: 9783319101699

[10] KARMAKAR, N. C., ZOMORRODI, M., DIVARATHNE, C. Advanced Chipless RFID. Hoboken (USA): Wiley, 2016. ISBN: 9781119-227311

[11] COSTA, F., GENOVESI, S., MONORCHIO, A. A Chipless RFID based on multiresonant high-impedance surfaces. IEEE Transactions on Microwave Theory and Techniques, 2013, vol. 61, no. 1, p. 146-153. DOI: 10.1109/TMTT.2012.2227777

[12] PLESSKY, V. P., REINDL, L. M. Review on SAW RFID tags. IEEE Transactions on Ultrasonics, Ferroelectrics, and Frequency Control, 2010, vol. 57, no. 3, p. 654-668. DOI: 10.1109/tuffc.2010.1462

[13] VENA, A., PERRET, E., TEDJINI, S. Design rules for chipless RFID tags based on multiple scatterers. Annals of Telecommunications, 2013, vol. 68, no. 7, p. 361-374. DOI: 10.1007/s12243-013-0358-7

[14] ZHONG, Y. Y., CHEN, Z. J., WANG, X. C., et al. Anti-collision SAW tags based on CIDT. In Proceedings of the Symposium on Piezoelectricity, Acoustic Waves, and Device Applications (SPAWDA). Jinan (China), 2015, p. 137-142. DOI: 10.1109/SPAWDA.2015.7364458
[15] RIAZ, M. A., SHAHID, H., ASLAM, S. Z., et al. Novel T-shaped resonator based chipless RFID tag. IEICE Electronics Express, 2017, vol. 14, no. 18, p. 20170728-20170728. DOI: $10.1587 /$ elex.14.20170728

[16] CHAMARTI, A., VARAHRAMYAN, K. Transmission delay line based ID generation circuit for RFID applications. IEEE Microwave and Wireless Components Letters, 2006, vol. 16, no. 11, p. 588-590. DOI: 10.1109/LMWC.2006.884897

[17] SCHUBLER, M., MANDEL, C., MAASCH, M., et al. Phase modulation scheme for chipless RFID-and wireless sensor tags. In Proceedings of the Asia Pacific Microwave Conference. Singapore, 2009, p. 229-232. DOI: 10.1109/APMC.2009.5385319

[18] ZHENG, L., RODRIGUEZ, S., ZHANG, L., et al. Design and implementation of a fully reconfigurable chipless RFID tag using Inkjet printing technology. In Proceedings of the IEEE International Symposium on Circuits and Systems. Seattle (USA), 2008, p. 1524-1527. DOI: $10.1109 /$ ISCAS.2008.4541720

[19] SUMI, M., DINESH, R., NIJAS, C. M., et al. Frequency coded chipless RFID tag using spurline resonators. Radioengineering, 2014, vol. 23, no. 1, p. 203-208. ISSN: 1805-9600

[20] RAUF, S., RIAZ, M. A., SHAHID, H., et al. Triangular loop resonator based compact chipless RFID tag. IEICE Electronics Express, 2017, vol. 14, no. 4, p. 20161262-20161262. DOI: $10.1587 /$ elex.14.20161262

[21] MACHAC, J., POLIVKA, M. Influence of mutual coupling on performance of small scatterers for chipless RFID tags. In Proceedings of the 24th International Conference Radioelektronika. Bratislava (Slovakia), 2014, p. 1-4. DOI: 10.1109/Radioelek.2014.6828412

[22] KHAN, M. M., TAHIR, F. A., CHEEMA, H. M. Frequency band utilization enhancement for chipless RFID tag through place value encoding. In Proceedings of the IEEE International Symposium on Antennas and Propagation (APSURSI). Fajardo (Puerto Rico), 2016, p. 1477-1478. DOI: 10.1109/APS.2016.7696445

[23] ISLAM, M. A., KARMAKAR, N. A compact printable dualpolarized chipless RFID tag using slot length variation in 'I' slot resonators. In Proceedings of the European Microwave Conference (EuMC). Paris (France), 2015, p. 96-99. DOI: 10.1109/EuMC.2015.7345708

[24] IQBAL, M. S., SHAHID, H., RIAZ, M. A., et al. FSS inspired polarization insensitive chipless RFID tag. IEICE Electronics Express, 2017, vol. 14, no. 10, p. 1-6. DOI: 10.1587/elex.14.20170243

[25] VENA, A., PERRET, E., TEDJINI, S. Chipless RFID tag using hybrid coding technique. IEEE Transactions on Microwave Theory and Techniques, 2011, vol. 59, no. 12, p. 3356-3364. DOI: 10.1109/TMTT.2011.2171001

[26] MARTINEZ, M., VAN DER WEIDE, D. Compact slot-based chipless RFID tag. In Proceedings of the IEEE RFID Technology and Applications Conference (RFID-TA). Tampere, (Finland), 2014, p. 233-236. DOI: 10.1109/RFID-TA.2014.6934234

\section{About the Authors...}

Muhammad Ali RIAZ received his M.S. and B.S. degree in Electrical Engineering from Iowa State University in 2010 and 2009 respectively. From January 2009 to December 2010 he was a Research Assistant with the Department of Electrical and Computer Engineering, Iowa State University. He is currently an Assistant Professor and a parttime Doctoral Researcher with ACTSENA research group at University of Engineering and Technology, Taxila. 
Yassin ABDULLAH received his B.S. degree in Telecommunication Engineering from Taiz University, Yemen in 2012. He got scholarship for the Masters in University and Engineering Technology, Taxila. He is currently working towards the M.Sc. degree and his research interest fields are signal processing and chipless RFID Technology.

Humayun SHAHID received his B.S. degree in Communication Systems Engineering from Institute of Space Technology, Islamabad in the year 2008, and his M.S. degree in Signal Processing from Nanyang Technological University, Singapore in the year 2011. His current research interests include antenna design, microwave engineering and RFID technology. He currently serves as an Assistant Professor at the Telecommunication Engineering Department, University of Engineering and Technology, Taxila.

Yasar AMIN is a Chairman and Associate Professor of the Telecommunication Engineering Department, University of Engineering and Technology, Taxila, Pakistan. He is the founder of ACTSENA Research Group at UET Taxila, Pakistan. He did his B.Sc. in Electrical Engineering in 2001 with specialization in Telecommunication, and M.Sc. in Electrical Engineering in 2003 with specialization in System-on-Chip Design from the Royal Institute of Technology (KTH), Sweden. His Ph.D. is in Electronic and Computer Systems from the Royal Institute of Technology (KTH), Sweden, with the research focus on printable green RFID antennas for embedded sensors, while he has MBA in Innovation and Growth from Turku School of Economics, University of Turku, Finland. He has done several specialized courses from Stanford University, California, USA and Massachusetts Institute of Technology (MIT), USA. He has supervised over 15 M.Sc. thesis, and presently supervising 8 doctoral thesis. $\mathrm{He}$ is presently serving as a leading Guest Editor at two international journals and an active reviewer of more than a dozen well reputed international journals. He has contributed to over 20 journal papers, over 30 reviewed international conference papers. Dr. Yasar is a member of IEEE, IET, ACM and ACES.

Adeel AKRAM received his B.S. degree in Electrical Engineering from the University of Engineering and Technology, Lahore, Pakistan in 1995. He received his M.S. degree in Computer Engineering from the National University of Sciences and Technology (NUST), Pakistan and his Ph.D. in Electrical Engineering from the University of Engineering and Technology (UET) Taxila, Pakistan, in 2000 and 2007 respectively. He is the Dean of Faculty of Telecommunication and Information Engineering at UET Taxila. His research interests include microwave and communication systems and is leading a $5 \mathrm{G}$ Wireless Communication research group at UET Taxila, Pakistan.

Hannu TENHUNEN is a chair professor of Electronic Systems at Royal Institute of Technology (KTH), Stockholm, Sweden. Prof. Tenhunen has held professor position as full professor, invited professor or visiting honorary professor in Finland (TUT, UTU), Sweden (KTH), USA (Cornel U), France (INPG), China (Fudan and Beijing Jiaotong Universities), and Hong Kong (Chinese University of Hong Kong), and has an honorary doctorate from Tallinn Technical University. He has been the director of multiple national large-scale research programs or being an initiator and director of national or Knowledge and Innovation Community EIT ICT Labs. European graduate schools. He has actively contributed to VLSI and SoC design in Finland and Sweden via creating new educational programs and research directions, most lately at European level as being the EU-level Education Director of the new European flagship initiative European Institute of Technology and Innovations (EIT), and its Knowledge and Innovation Community EIT ICT Labs. 